\title{
SECTIONAL CURVATURES OF GEODESIC SPHERES IN A COMPLEX HYPERBOLIC SPACE
}

\author{
Tetsuo Kajiwara and Sadahiro Maeda \\ Dedicated to Professor Hiroshi Asano on the occasion of his 80th birthday
}

\begin{abstract}
We characterize geodesic spheres with sufficiently small radii in a complex hyperbolic space of constant holomorphic sectional curvature $c(<0)$ by using their geometric three properties. These properties are based on their contact forms, geodesics and shape operators. These geodesic spheres are the only examples of hypersurfaces of type (A) which are of nonnegative sectional curvature in this ambient space. Moreover, in particular, when $-1 \leqq c<0$, the class of these geodesic spheres has just one example of Sasakian space forms.
\end{abstract}

\section{Introduction}

We denote by $\tilde{M}_{n}(c)$ a complex $n$-dimensional complete and simply connected Kähler manifold of constant holomorphic sectional curvature $c(\neq 0)$, namely it is holomorphically isometric to either an $n$-dimensional complex projective space $\mathbf{C} P^{n}(c)$ of constant holomorphic sectional curvature $c$ or an $n$-dimensional complex hyperbolic space $\mathbf{C H}^{n}(c)$ of constant holomorphic sectional curvature $c$ according as $c$ is positive or negative, which is called an $n$-dimensional nonflat complex space form of constant holomorphic sectional curvature $c$.

In the theory of real hypersurfaces $M^{2 n-1}$ isometrically immersed into $\tilde{M}_{n}(c)$, hypersurfaces of type (A) are the most important examples (see Section 2).

When $c>0$, every hypersurface of type (A) is of nonnegative sectional curvature (see Proposition A). On the contrary, the horosphere, which is a

2010 Mathematics Subject Classification. Primary 53B25, Secondary 53C40.

Key words and phrases. Geodesic spheres, complex hyperbolic spaces, sectional curvatures, exterior differentiation, contact form, geodesics, circles, shape operator, strongly $\phi$-invariant, weakly $\phi$-invariant, Hopf hypersurfaces, hypersurfaces of type (A), Sasakian space forms.

The second author is partially supported by Grant-in-Aid for Scientific Research (C) (No. 23540097), Japan Society for the Promotion of Sciences.

Received September 26, 2014; revised March 3, 2015. 
typical example of hypersurfaces of type (A) in $\mathbf{C H}(c)$, has the sectional curvature $K$ with $3 c / 4 \leqq K \leqq-c / 4$. Motivated by this fact, we are interested in nonnegatively curved hypersurfaces of type (A) in $\mathbf{C} H^{n}(c)$.

We shall classify nonnegatively curved hypersurfaces $M^{2 n-1}$ of type (A) in $\mathrm{CH}^{n}(c)$ (cf. Remark 3(3)) and characterize them in terms of the extrinsic shape of some geodesics of $M$, the exterior differentiation $d \eta$ of the contact form $\eta$ on $M$ and the weakly $\phi$-invariance of the shape operator $A$ of $M$ in this ambient space (see Theorems 1, 2 and 3).

Our motivation is mainly based on the following three facts on real hypersurfaces $M$.

(1) There exist no real hypersurfaces $M$ all of whose geodesics are mapped to circles in a nonflat complex space form $\tilde{M}_{n}(c), n \geqq 2$.

(2) There exist no real hypersurfaces $M$ with closed contact form $\eta$, namely there does not exist an open (non-empty) subset $U$ on each real hypersurface $M$ in a nonflat complex space form $\tilde{M}_{n}(c), n \geqq 2$ such that $d \eta=0$ on $U$.

(3) There exist no real hypersurfaces $M$ with strongly $\phi$-invariant shape operator $A$ in $\mathbf{C} H^{n}(c), n \geqq 2$, that is, there does not exist $M$ satisfying that $g(A \phi X, \phi Y)=g(A X, Y)$ for all vectors $X, Y$ on $M$ in the ambient space $\mathbf{C} H^{n}(c)$.

Here, $(\phi, \xi, \eta, g)$ is the almost contact metric structure on $M$ induced from the Kähler structure $(J, g)$ of the ambient space $\tilde{M}_{n}(c), n \geqq 2$.

Inspired by the above three Facts (1), (2) and (3), we shall prove the following three theorems.

THEOREM 1. A connected real hypersurface $M^{2 n-1}$ isometrically immersed into $\mathbf{C} H^{n}(c), n \geqq 2$ is locally congruent to a geodesic sphere $G(r)$ of radius $r$ all of whose sectional curvatures are nonnegative if and only if at every point $p$ of $M$, there exists a positive constant $k(p)$ with $k(p) \geqq \sqrt{|c|}$ such that all geodesics $\gamma_{i}=\gamma_{i}(s)$ on $M$ with $\gamma_{i}(0)=p, \dot{\gamma}_{i}(0)=v_{i}(1 \leqq i \leqq 2 n-2)$ are mapped to circles of the same curvature $k(p)$ in the ambient space $\mathbf{C} H^{n}(c)$ for some orthonormal vectors $v_{1}, \ldots, v_{2 n-2}\left(\in T_{p} M\right)$ orthogonal to the characteristic vector $\xi_{p}$. Here, the function $k=k(p)$ is automatically constant on $M$.

We note that all sectional curvatures of a geodesic sphere $G(r)$ are nonnegative if and only if its radius $r$ satisfies $0<r \leqq \log 3 / \sqrt{|c|}$.

THEOREM 2. A connected real hypersurface $M^{2 n-1}$ isometrically immersed into $\mathbf{C} H^{n}(c), n \geqq 2$ is locally congruent to a geodesic sphere $G(r)$ of radius $r$ with $0<r \leqq \log 3 / \sqrt{|c|}$ if and only if it satisfies the following conditions:

(1) There exists a positive constant $k$ such that $M$ satisfies either $d \eta(X, Y)=$ $k g(X, \phi Y)$ for all $X, Y \in T M$ or $d \eta(X, Y)=-k g(X, \phi Y)$ for all $X, Y \in$ $T M$

(2) There exists a point $x \in M$ where $M$ is nonnegatively curved. 
In Theorems 1 and $2, k$ is expressed as: $k=(\sqrt{|c|} / 2) \operatorname{coth}(\sqrt{|c|} r / 2)$, so that $k \geqq \sqrt{|c|}$ and the radius $r$ of $G(r)$ is given by $r=(1 / \sqrt{|c|})\{\log (2 k+\sqrt{|c|})-$ $\log (2 k-\sqrt{|c|})\}$.

THEOREM 3. A connected real hypersurface $M^{2 n-1}$ isometrically immersed into $\mathbf{C} H^{n}(c), n \geqq 2$ is locally congruent to a geodesic sphere $G(r)$ of radius $r$ with $0<r \leqq \log 3 / \sqrt{|c|}$ if and only if it satisfies the following conditions:

(1) $M$ is a Hopf hypersurface with weakly $\phi$-invariant shape operator A;

(2) There exists a point $x \in M$ where $M$ is nonnegatively curved.

In Theorem 3, the condition that the shape operator $A$ of $M$ is weakly $\phi$-invariant means that $g(A \phi X, \phi Y)=g(A X, Y)$ holds for all vectors $X$ and $Y$ orthogonal to the characteristic vector field $\xi$ of $M$.

Theorem 1 gives a geometric meaning of Theorems 2 and 3. Related to our geodesic spheres in our Theorems, from the viewpoint of contact geometry we shall prove the following:

Proposition 1. When $-1 \leqq c<0$, the class of geodesic spheres $G(r)$ of radius $r$ with $0<r \leqq \log 3 / \sqrt{|c|}$ in $\mathbf{C} H^{n}(c), n \geqq 2$ has just one example of Sasakian space forms. In this case, it has automatically constant $\phi$-sectional curvature $c+1$ and is a geodesic sphere of radius $r$ with $\tanh (\sqrt{|c|} r / 2)=\sqrt{|c|} / 2$.

The authors would like to express their hearty thanks to the referee for kind advices.

\section{Preliminaries}

Let $M^{2 n-1}$ be a real hypersurface with a unit normal local vector field $\mathscr{N}$ of an $n(\geqq 2)$-dimensional nonflat complex space form $\tilde{M}_{n}(c)$ through an isometric immersion. The ambient space $\tilde{M}_{n}(c)$ is furnished with the standard Riemannian metric $g$ and the canonical Kähler structure $J$. The Riemannian connections $\tilde{\nabla}$ of $\tilde{M}_{n}(c)$ and $\nabla$ of $M$ are related by the following formulas of Gauss and Weingarten:

$$
\begin{gathered}
\tilde{\nabla}_{X} Y=\nabla_{X} Y+g(A X, Y) \mathscr{N}, \\
\tilde{\nabla}_{X} \mathscr{N}=-A X
\end{gathered}
$$

for arbitrary vector fields $X$ and $Y$ on $M$, where $g$ is the Riemannian metric of $M$ induced from the standard metric of the ambient space $\tilde{M}_{n}(c)$ and $A$ is the shape operator of $M$ in $\tilde{M}_{n}(c)$. An eigenvector of the shape operator $A$ is called a principal curvature vector of $M$ in $\tilde{M}_{n}(c)$ and an eigenvalue of $A$ is called a principal curvature of $M$ in $\tilde{M}_{n}(c)$. We denote by $V_{\lambda}$ the eigenspace associated with the principal curvature $\lambda$, namely we set $V_{\lambda}=\{v \in T M \mid A v=\lambda v\}$. 
On $M$ it is well-known that an almost contact metric structure $(\phi, \xi, \eta, g)$ associated with $\mathscr{N}$ is canonically induced from the Kähler structure $(J, g)$ of the ambient space $\tilde{M}_{n}(c)$, which is defined by

$$
g(\phi X, Y)=g(J X, Y), \quad \xi=-J \mathscr{N} \quad \text { and } \quad \eta(X)=g(\xi, X)=g(J X, \mathscr{N}) .
$$

It follows from (2.1), (2.2) and $\tilde{\nabla} J=0$ that

$$
\begin{gathered}
\left(\nabla_{X} \phi\right) Y=\eta(Y) A X-g(A X, Y) \xi \\
\nabla_{X} \xi=\phi A X .
\end{gathered}
$$

In general, changing $\mathscr{N}$ into $-\mathscr{N}$, we have two almost contact metric structures $(\phi, \xi, \eta, g)$ and $(\phi,-\xi,-\eta, g)$ to each real hypersurface $M$ in $\tilde{M}_{n}(c)$. The following is called the equation of Codazzi:

$$
\left(\nabla_{X} A\right) Y-\left(\nabla_{Y} A\right) X=(c / 4)(\eta(X) \phi Y-\eta(Y) \phi X-2 g(\phi X, Y) \xi) .
$$

Denoting the curvature tensor of $M$ by $R$, we have the equation of Gauss given by

$$
\begin{aligned}
g((R(X, Y) Z, W)=(c / 4)\{ & g(Y, Z) g(X, W)-g(X, Z) g(Y, W) \\
& +g(\phi Y, Z) g(\phi X, W)-g(\phi X, Z) g(\phi Y, W) \\
& -2 g(\phi X, Y) g(\phi Z, W)\} \\
+ & g(A Y, Z) g(A X, W)-g(A X, Z) g(A Y, W) .
\end{aligned}
$$

Hence, the sectional curvature $K(X, Y)$ of the real plane spanned by a pair $(X, Y)$ of orthonormal vectors is given by

$$
K(X, Y)=(c / 4)\left(1+3 g(\phi X, Y)^{2}\right)+g(A X, X) g(A Y, Y)-g(A X, Y)^{2} .
$$

We usually call $M$ a Hopf hypersurface if the characteristic vector $\xi$ is a principal curvature vector at each point of $M$. Note that every tube of a sufficiently small constant radius around each Kähler submanifold of $\tilde{M}_{n}(c)$ is a Hopf hypersurface.

LeMma A $([12,9])$. Let $M$ be a Hopf hypsurface of a nonflat complex space form $\tilde{M}_{n}(c), n \geqq 2$. Then the following hold.

(1) If a nonzero vector $v \in T M$ orthogonal to $\xi$ satisfies $A v=\lambda v$, then $(2 \lambda-\delta) A \phi v=(\delta \lambda+(c / 2)) \phi v$, where $\delta$ is the principal curvature associated with $\xi$. In particular, when $c>0$, we have $A \phi v=((\delta \lambda+(c / 2)) /$ $(2 \lambda-\delta)) \phi v$.

(2) The principal curvature $\delta$ associated with $\xi$ is constant locally.

We recall the following real hypersurfaces which are the simplest examples of Hopf hypersurfaces. 
When $c>0$,

$\left(A_{1}\right)$ a geodesic sphere $G(r)$ of radius $r(0<r<\pi / \sqrt{c})$ in $\mathbf{C} P^{n}(c)$,

$\left(A_{2}\right)$ a tube of radius $r(0<r<\pi / \sqrt{c})$ around a totally geodesic complex submanifold $\mathbf{C} P^{\ell}(c)$ with $1 \leqq \ell \leqq n-2$ in $\mathbf{C} P^{n}(c)$.

When $c<0$,

$\left(A_{0}\right)$ a horosphere $H S$ in $\mathbf{C H}^{n}(c)$,

$\left(A_{1,0}\right)$ a geodesic sphere $G(r)$ of radius $r(0<r<\infty)$ in $\mathbf{C H}^{n}(c)$,

$\left(A_{1,1}\right)$ a tube of radius $r(0<r<\infty)$ around a totally geodesic complex hypersurface $\mathrm{CH}^{n-1}(c)$ in $\mathbf{C} H^{n}(c)$,

$\left(A_{2}\right)$ a tube of radius $r(0<r<\infty)$ around a totally geodesic complex submanifold $\mathrm{CH}^{\ell}(c)$ with $1 \leqq \ell \leqq n-2$.

Unifying these real hypersurfaces in $\tilde{M}_{n}(c), n \geqq 2$, we call them hypersurfaces of type (A). The following shows the importance of hypersurfaces of type (A) in the theory of real hypersurfaces in $\tilde{M}_{n}(c)$ (for example, see $[12,13]$ ).

THEOREM A. For every real hyersurface $M$ in a nonflat complex space form $\tilde{M}_{n}(c), n \geqq 2$, the length of the derivative of the shape operator $A$ of $M$ satisfies $\|\nabla A\|^{2} \geqq\left(c^{2} / 4\right)(n-1)>0$ at its each point. In particular, $\|\nabla A\|^{2}=\left(c^{2} / 4\right)(n-1)$ holds on $M$ if and only if $M$ is locally congruent to a hypersurface of type (A).

The following is a well-known characterization of hypersurfaces of type (A) in $\tilde{M}_{n}(c)$ (cf. [13]).

LEMma B. Let $M$ be a connected real hypersurface in a nonflat complex space form $\tilde{M}_{n}(c), n \geqq 2$. Then $M$ is locally congruent to a hypersurface of type (A) if and only if $\phi A=A \phi$ holds on $M$, where $A$ is the shape operator of $M$ in this ambient space and $\phi$ is the structure tensor on $M$.

It is well-known that every hypersurface of type (A) is a homogeneous real hypersurface in $\tilde{M}_{n}(c)$, namely it is an orbit of some subgroup of the isometry group $\mathrm{I}\left(\tilde{M}_{n}(c)\right)$ of the ambient space $\tilde{M}_{n}(c)$.

The following gives information on sectional curvatures of all hypersurfaces of type (A) in $\mathbf{C} P^{n}(c)$ (for example, see [8]).

Proposition A. The sectional curvature $K$ of hypersurfaces of type (A) with radius $r(0<r<\pi / \sqrt{c})$ in $\mathbf{C} P^{n}(c), n \geqq 2$ satisfies the following inequalities:

$\left(\mathrm{A}_{1}\right)(c / 4) \cot ^{2}(\sqrt{c} r / 2) \leqq K \leqq c+(c / 4) \cot ^{2}(\sqrt{c} r / 2)$;

$\left(\mathrm{A}_{2}\right) 0 \leqq K \leqq c+(c / 4) \max \left\{\cot ^{2}(\sqrt{c} r / 2), \tan ^{2}(\sqrt{c} r / 2)\right\}$.

Note that these estimations in Proposition A are sharp.

For the later use we recall the classification theorem of homogeneous Hopf hypersurfaces in $\mathrm{CH}^{n}(c)$.

TheOREM B ([4]). Let $M^{2 n-1}$ be a connected Hopf hypersurface of $\mathbf{C} H^{n}(c)$, $n \geqq 2$. Then all of the principal curvatures of $M$ are constant if and only if $M$ is 
locally congruent to either a homogeneous real hypersurface of type (A) (i.e., a hypersurface of type (A)) or a homogeneous real hypersurface of type (B) which is a tube of radius $r(0<r<\infty)$ around a totally real totally geodesic $\mathbf{R} H^{n}(c / 4)$ which is the real part of $\mathbf{C} H^{n}(c)$.

Remark 1. Every homogeneous real hypersurface in $\mathbf{C} P^{n}(c)$ is a Hopf hypersurface. But, in $\mathrm{CH}^{n}(c)$ there exist many homogeneous non-Hopf hypersurfaces as well as many homogeneous Hopf hypersurfaces (cf. [14, 5]).

A homogeneous real hypersurface of type (B) with radius $r=$ $(1 / \sqrt{|c|}) \log _{e}(2+\sqrt{3})$ has two distinct constant principal curvatures $\lambda_{1}=\delta=$ $\sqrt{3|c|} / 2$ and $\lambda_{2}=\sqrt{|c|} /(2 \sqrt{3})$. Except for this real hypersurface, the numbers of distinct principal curvatures of homogeneous Hopf hypersurfaces are 2, 2, 2, 3, 3, respectively. The principal curvatures of these real hypersurfaces in $\mathbf{C} H^{n}(c)$ are given as follows (see [4]):

\begin{tabular}{|c|c|c|c|c|c|}
\hline & $\left(\mathrm{A}_{0}\right)$ & $\left(\mathrm{A}_{1,0}\right)$ & $\left(\mathrm{A}_{1,1}\right)$ & $\left(\mathrm{A}_{2}\right)$ & $(\mathrm{B})$ \\
\hline$\lambda_{1}$ & $\frac{\sqrt{|c|}}{2}$ & $\frac{\sqrt{|c|}}{2} \operatorname{coth}\left(\frac{\sqrt{|c|}}{2} r\right)$ & $\frac{\sqrt{|c|}}{2} \tanh \left(\frac{\sqrt{|c|}}{2} r\right)$ & $\frac{\sqrt{|c|}}{2} \operatorname{coth}\left(\frac{\sqrt{|c|}}{2} r\right)$ & $\frac{\sqrt{|c|}}{2} \operatorname{coth}\left(\frac{\sqrt{|c|}}{2} r\right)$ \\
\hline$\lambda_{2}$ & - & - & - & $\frac{\sqrt{|c|}}{2} \tanh \left(\frac{\sqrt{|c|}}{2} r\right)$ & $\frac{\sqrt{|c|}}{2} \tanh \left(\frac{\sqrt{|c|}}{2} r\right)$ \\
\hline$\delta$ & $\sqrt{|c|}$ & $\sqrt{|c|} \operatorname{coth}(\sqrt{|c|} r)$ & $\sqrt{|c|} \operatorname{coth}(\sqrt{|c|} r)$ & $\sqrt{|c|} \operatorname{coth}(\sqrt{|c|} r)$ & $\sqrt{|c|} \tanh (\sqrt{|c|} r)$ \\
\hline
\end{tabular}

\section{Real hypersurfaces some of whose geodesics are mapped to circles in a complex hyperbolic space}

First of all we explain the background of Fact (1) in Introduction. We review the definition of circles in Riemannian geometry. A smooth real curve $\gamma=\gamma(s)$ parametrized by its arclength $s$ on a Riemannian manifold $M$ with Riemannian connection $\nabla$ is called a circle of curvature $k$ if there exist a nonnegative constant $k$ and the unit vector field $Y_{s}$ orthogonal to the tangential vector $\dot{\gamma}$ along the curve $\gamma$ satisfying the ordinary differential equations $\nabla_{\dot{\gamma}} \dot{\gamma}=k Y_{s}$ and $\nabla_{\dot{\gamma}} Y_{s}=-k \dot{\gamma}$. It is well-known that a curve $\gamma$ is a circle if and only if it satisfies the following differential equation:

$$
\nabla_{\dot{\gamma}} \nabla_{\dot{\gamma}} \dot{\gamma}+g\left(\nabla_{\dot{\gamma}} \dot{\gamma}, \nabla_{\dot{\gamma}} \dot{\gamma}\right) \dot{\gamma}=0
$$

where $g$ is the Riemannian metric on $M$. A circle of null curvature is nothing but a geodesic. The following is fundamental in the theory of hypersurfaces:

Proposition 2. For a connected hypersurface $M^{n}$ isometrically immersed into a Riemannian manifold $\tilde{M}^{n+1}$ the following three conditions are mutually equivalent. 
(1) Every geodesic $\gamma$ on $M^{n}$ is mapped to a circle in $\tilde{M}^{n+1}$.

(2) Every geodesic $\gamma$ on $M^{n}$ is mapped to a circle of the same curvature which is independent of the choice of $\gamma$ in $\tilde{M}^{n+1}$.

(3) $M^{n}$ is totally umbilic in $\tilde{M}^{n+1}$ and Trace $A$ is constant locally on $M^{n}$, where $A$ is the shape operator of $M^{n}$ in $\tilde{M}^{n+1}$.

Proof. We suppose Condition (1). Then, from (3.1) every geodesic $\gamma$ of $M^{n}$, considered as a curve in the ambient space $\tilde{M}^{n+1}$, satisfies the following ordinary differential equation:

$$
\tilde{\nabla}_{\dot{\gamma}} \tilde{\nabla}_{\dot{\gamma}} \dot{\gamma}+g\left(\tilde{\nabla}_{\dot{\gamma}} \dot{\gamma}, \tilde{\nabla}_{\dot{\gamma}} \dot{\gamma}\right) \dot{\gamma}=0 .
$$

On the other hand, in consideration of Gauss formula: $\tilde{\nabla}_{X} Y=\nabla_{X} Y+$ $g(A X, Y) \mathscr{N}$ and Weingarten formula: $\tilde{\nabla}_{X} \mathscr{N}=-A X$ for the hypersurface $M^{n}$ in $\tilde{M}^{n+1}$, we can rewrite (3.2) as follows:

$$
-g(A \dot{\gamma}, \dot{\gamma}) A \dot{\gamma}+g(A \dot{\gamma}, \dot{\gamma})^{2} \dot{\gamma}+g\left(\left(\nabla_{\dot{\gamma}} A\right) \dot{\gamma}, \dot{\gamma}\right) \mathscr{N}=0 .
$$

Hence, taking the tangential component and the normal component of (3.3) for the hypersurace $M^{n}$ in $\tilde{M}^{n+1}$, we obtain

$$
g(A \dot{\gamma}, \dot{\gamma}) A \dot{\gamma}=g(A \dot{\gamma}, \dot{\gamma})^{2} \dot{\gamma} \quad \text { and } \quad g\left(\left(\nabla_{\dot{\gamma}} A\right) \dot{\gamma}, \dot{\gamma}\right)=0
$$

for each geodesic $\gamma$ on $M^{n}$. Equation (3.4) means that

$$
g(A X, X) A X=g(A X, X)^{2} X \quad \text { and } \quad g\left(\left(\nabla_{X} A\right) X, X\right)=0
$$

for all $X \in T M$ with $\|X\|=1$. Note that the former equation in (3.5) means

$$
g(A X, X) g(A X, Y)=0
$$

for each pair of orthonormal vectors $X$ and $Y$ on $M$, which is equivalent to saying that

$$
g\left(A_{p} X, X\right)^{2} \text { is constant at each point } p \in M
$$

for every unit vector $X \in T_{p} M$.

Indeed, let $f: S^{n-1}(1)\left(\subset \mathbf{R}^{n}\right) \rightarrow \mathbf{R}$ be the differentiable function on a subset $S^{n-1}(1) \cong\left\{u \in T_{p} M \mid\|u\|=1\right\}$ defined by $f(u)=g\left(A_{p} u, u\right)^{2}$, where $A_{p}$ is the shape operator of $M$ in $\tilde{M}^{n+1}$ at the point $p \in M$. If $v$ is a vector tangent to $S^{n-1}(1)$ at $u$ (hence $u \perp v$ ), we find $v(f)=4 g\left(A_{p} u, u\right) g\left(A_{p} u, v\right)=0$ by (3.6). Thus $f$ is a constant function on $S^{n-1}(1)$.

Then we can set $\lambda^{2}(p)=g(A X, X)^{2}$ for each unit vector $X \in T_{p} M$ with $\lambda(p) \geqq 0$ at every point $p \in M$. When $M^{n}$ is not totally geodesic in $\tilde{M}^{n+1}$, there exists a point $x \in M$ with $\lambda(x)>0$. Then the continuity of the function $\lambda$ shows that there exists some open neighborhood $U_{x}$ of the point $x$ such that $\lambda>0$ on $U_{x}$. We here choose a local field of orthonormal frames $e_{1}, \ldots, e_{n}$ on $U_{x}$ in such 
a way that $A e_{i}=\lambda_{i} e_{i}(1 \leqq i \leqq n)$. Hence, from (3.7) we see that $\lambda_{1}^{2}=\cdots=$ $\lambda_{n}^{2}=\lambda^{2}$. In this case, we suppose that there exist an orthonormal pair of vectors $e_{i}$ and $e_{j}$ such that $A e_{i}=\lambda e_{i}$ and $A e_{j}=-\lambda e_{j}$. Then we find that

$$
g\left(A\left(e_{i}+e_{j}\right) / \sqrt{2},\left(e_{i}+e_{j}\right) / \sqrt{2}\right)=0,
$$

which is a contradiction. So, we know that either $A e_{i}=\lambda e_{i}(1 \leqq i \leqq n)$ or $A e_{i}=$ $-\lambda e_{i}(1 \leqq i \leqq n)$, which shows that every point $y \in U_{x}$ is an umbilic point. Thus we can see that $M^{n}$ is totally umbilic in $\tilde{M}^{n+1}$. Furthermore, the latter equation in (3.5) yields that the function $\lambda$ is constant locally on $M$. Therefore we get Conditions (2) and (3) in our Proposition. In this case, note that every geodesic $\gamma$ on $M^{n}$ is mapped to a circle of the same curvature $\lambda(\geqq 0)$ which is independent of the choice of $\gamma$.

Conversely, we suppose Condition (3). Then we have Equation (3.5). Hence by (3.2) we obtain Condition (1).

The following is well-known:

Proposition 3 ([15]). There exist no totally umbilic real hypersurfaces in a nonflat complex space form $\tilde{M}_{n}(c), n \geqq 2$.

Proof. Suppose that $A X=\lambda X$ for all vectors $X$ on $M$. For any vectors $X$ and $Y$ orthogonal to $\xi$, from the Codazzi equation (2.5) we have $(X \lambda) Y-$ $(Y \lambda) X=(c / 2) g(X, \phi Y) \xi$, so that $g(X, \phi Y)=0$ for all $X, Y$ perpendicular to $\xi$. This is a contradiction.

By virtue of Propositions 2 and 3 we obtain Fact (1).

We shall prove Theorem 1.

$(\Rightarrow)$ First of all we remark that a geodesic sphere $G(r)(0<r<\infty)$ in $\mathrm{CH}^{n}(c)$ is of nonnegative sectional curvature if and only if the radius $r$ satisfies $0<r \leqq \log 3 / \sqrt{|c|}$ (for example, see the proof of Theorem 2).

Let $M$ be a geodesic sphere $G(r)$ of radius $r$ all of whose sectional curvatures are nonnegative in the ambient space $\mathbf{C} H^{n}(c)$. Let $\gamma=\gamma(s)$ be an arbitrary geodesic with $\gamma(0)=p$ on our real hypersurface $M$ whose initial vector $\dot{\gamma}(0)$ is perpendicular to the characteristic vector $\xi_{p}$. It follows from (2.4), Lemma B, the symmetry of $A$ and the skew-symmetry of $\phi$ that

$$
\begin{aligned}
\dot{\gamma}(g(\dot{\gamma}, \xi)) & =g\left(\dot{\gamma}, \nabla_{\dot{\gamma}} \xi\right)=g(\dot{\gamma}, \phi A \dot{\gamma})=g(\dot{\gamma}, A \phi \dot{\gamma}) \\
& =g(A \dot{\gamma}, \phi \dot{\gamma})=-g(\phi A \dot{\gamma}, \dot{\gamma})=0,
\end{aligned}
$$

which, combined with $g\left(\dot{\gamma}(0), \xi_{\gamma(0)}\right)=0$, shows that $\dot{\gamma}(s)$ is orthogonal to $\xi_{\gamma(s)}$ for every $s$. Thus we have $A \dot{\gamma}(s)=(\sqrt{|c|} / 2) \operatorname{coth}(\sqrt{|c|} r / 2) \dot{\gamma}(s)$ for $-\infty<s<\infty$. This, together with (2.1) and (2.2), implies that the geodesic $\gamma$ is mapped to a circle of the same positive curvature $(\sqrt{|c|} / 2) \operatorname{coth}(\sqrt{|c|} r / 2)$ in $\mathbf{C} H^{n}(c)$. Here, by the assumption $0<r \leqq \log 3 / \sqrt{|c|}$ we see $(\sqrt{|c|} / 2) \operatorname{coth}(\sqrt{|c|} r / 2) \geqq \sqrt{|c|}$. Thus we have proved the "only if" part in our Theorem. 
$(\Leftarrow)$ Let $\gamma_{i}=\gamma_{i}(s) \quad(1 \leqq i \leqq 2 n-2)$ be geodesics on $M$ satisfying the condition in the "if" part in our Theorem. Then it follows from (3.1) that

$$
\tilde{\nabla}_{\dot{\gamma}_{i}}\left(\tilde{\nabla}_{\dot{\gamma}_{i}} \dot{\gamma}_{i}\right)=-k^{2} \dot{\gamma}_{i}
$$

On the other hand, from (2.1) and (2.2) we obtain

$$
\tilde{\nabla}_{\dot{\gamma}_{i}}\left(\tilde{\nabla}_{\dot{\gamma}_{i}} \dot{\gamma}_{i}\right)=g\left(\left(\nabla_{\dot{\gamma}_{i}} A\right) \dot{\gamma}_{i}, \dot{\gamma}_{i}\right) \mathscr{N}-g\left(A \dot{\gamma}_{i}, \dot{\gamma}_{i}\right) A \dot{\gamma}_{i}
$$

Comparing the tangential components of (3.8) and (3.9), we have

$$
g\left(A \dot{\gamma}_{i}(s), \dot{\gamma}_{i}(s)\right) A \dot{\gamma}_{i}(s)=k^{2} \dot{\gamma}_{i}(s) \text { for } 1 \leqq i \leqq 2 n-2,
$$

which, combined with $k(p) \neq 0$, yields that $A v_{i}=k(p) v_{i}$ or $A v_{i}=-k(p) v_{i}$ for $1 \leqq i \leqq 2 n-2$ at the point $p=\gamma(0)$. Note that $\xi$ is principal. Indeed, $g\left(A \xi, v_{i}\right)$ $=g\left(\xi, A v_{i}\right)=0$ for $1 \leqq i \leqq 2 n-2$. Thus we know that our real hypersurface $M$ is a Hopf hypersurface having at most three distinct principal curvatures $\delta, k$ and $-k$. We here show that the function $k=k(p)$ is automatically constant on $M$.

We first consider the case of $2 k-\delta \neq 0$ at a point $x_{0} \in M$. Then by the continuity of this function it does not vanish on a sufficiently small neighborhood $U_{x_{0}}$ of the point $x_{0}$. This, together with Lemma $\mathrm{A}(1)$, yields

$$
k=\frac{k \delta+(c / 2)}{2 k-\delta} \quad \text { or } \quad k=-\frac{k \delta+(c / 2)}{2 k-\delta} .
$$

Hence $k$ is constant on the neighborhood $U_{x_{0}}$. We finally consider the case of $2 k-\delta=0$ at a point $x_{0} \in M$. We shall show that the function $2 k-\delta$ vanishes identically on some neighborhood $V_{x_{0}}$ of the point $x_{0}$. We here use reductio ad absurdum. Suppose that there does not exist a neighborhood of the point $x_{0}$ on which the function $2 k-\delta$ vanishes identically. Then there exists a point sequence $\left\{x_{n}\right\}$ on $M$ with $\lim _{n \rightarrow \infty} x_{n}=x_{0}$ and $(2 k-\delta)\left(x_{n}\right) \neq 0$ for each $n$. We note that the discussion in the case that $2 k-\delta \neq 0$ means that for each $n$ the function $2 k-\delta$ is nonzero constant on some sufficiently small neighborhood $V_{x_{n}}$ of the point $x_{n}$. This, together with the fact that every principal curvature of $M$ is continuous on $M$, shows that $2 k-\delta \neq 0$ at the point $x_{0}$ of $M$, which is a contradiction. Thus we can see that the function $k=k(p)$ is constant on $M$. Hence we find that the principal curvature $k$ is also constant locally in the case that $2 k-\delta=0$ at some point $x_{0}$ of $M$. Then we know that our real hypersurface $M$ is a Hopf hypersurface having at most three distinct constant principal curvatures $\delta, k$ and $-k$.

Therefore by the table of the principal curvatures of homogeneous Hopf hypersurfaces in $\mathbf{C H}^{n}(c)$ we find that our Hopf hypersurface $M$ has two distinct constant principal curvatures either $\delta, k$ or $\delta,-k$. Then without loss of generality $M$ has two distinct constant principal curvatures $\delta$ and $k(\geqq \sqrt{|c|})$. Therefore our discussion guarantees that our real hypersurface $M$ is locally congruent to a geodesic sphere $G(r)$ with $0<r \leqq \log 3 / \sqrt{|c|}$. Hence we have 
shown the "if" part in our Theorem. So we obtain the desired statement of Theorem 1.

The discussion in the proof of Theorem 1 yields the following:

Proposition 4. Every geodesic $\gamma$ on a geodesic sphere $G(r)(\log 3 / \sqrt{|c|}<$ $r<\infty)$ whose initial vector $\dot{\gamma}(0)$ is perpendicular to the characteristic vector $\xi_{\gamma(0)}$ is mapped to the same positive curvature $k$ with $\sqrt{|c|} / 2<k<\sqrt{|c|}$ in the ambient space $\mathbf{C} H^{n}(c), n \geqq 2$, where $k=(\sqrt{|c|} / 2) \operatorname{coth}(\sqrt{|c|} r / 2)$.

At the end of this section we explain the extrinsic shape $l \circ \gamma$ of every geodesic $\gamma$ on $G(r)(0<r<\infty)$ through the natural isometric embedding $l: G(r)$ $\rightarrow \mathbf{C} H^{n}(c)$ (for details, see Proposition 3.1 in [2]).

Proposition B. For a geodesic $\gamma$ on $G(r)(0<r<\infty)$ in $\mathbf{C} H^{n}(c), n \geqq 2$, through the inclusion mapping $l: G(r) \rightarrow \mathbf{C} H^{n}(c)$ the curve $l \circ \gamma$ is as follows:

(1) When the initial vector $\dot{\gamma}(0)$ is equal to $\xi_{\gamma(0)}, \imath \circ \gamma$ is a circle of positive curvature $\sqrt{|c|} \operatorname{coth}(\sqrt{|c|} r)$ on a complex line $\mathbf{C} H^{1}(c)$ in $\mathbf{C H}(c)$;

(2) When the initial vector $\dot{\gamma}(0)$ is orthogonal to $\xi_{\gamma(0)}, l \circ \gamma$ is a circle of positive curvature $(\sqrt{|c|} / 2) \operatorname{coth}(\sqrt{|c|} r / 2)$ on a totally real totally geodesic $\mathbf{R} H^{2}(c / 4)$ in $\mathbf{C} H^{n}(c)$;

(3) When the initial vector $\dot{\gamma}(0)$ is neither the form in (1) nor that in (2), $\iota \circ \gamma$ is a helix of proper order 4, namely it has three positive constant curvatures $k_{1}, k_{2}$ and $k_{3}$ and each of its other curvatures $k_{d}(d \geqq 4)$ vanishes in the sense of Frenet formula, on a totally geodesic complex submanifold $\mathbf{C} H^{2}(c)$ in $\mathbf{C} H^{n}(c)$.

\section{Exterior differentiation of the contact form on real hypersurfaces in a complex hyperbolic space}

Before proving Theorem 2 we explain the background of Fact (2) in Introduction. To do this, we first recall the definition of $d \eta$, which is given by

$$
d \eta(X, Y)=(1 / 2)\{X(\eta(Y))-Y(\eta(X))-\eta([X, Y])\} \text { for all } X, Y \in T M
$$

It follows from (2.4) and (4.1) that $d \eta=0$ if and only if $\phi A+A \phi=0$. This, together with the fact that there exist no real hypersurfaces $M$ with $\phi A+A \phi=0$ on $M$ (see Corollary 2. 12 in [13]), implies Fact (1).

We are now in a position to prove Theorem 2 .

$(\Leftarrow)$ It follows from $d \eta(X, Y)= \pm k g(X, \phi Y)$ for all $X, Y \in T M,(4.1)$ and (2.4) that

$$
0=g(\phi A X, Y)-g(\phi A Y, X) \mp 2 k g(X, \phi Y)=g((\phi A+A \phi \pm 2 k \phi) X, Y)
$$

for each $X, Y \in T M$. So our real hypersurface $M$ satisfies

$$
\phi A+A \phi=\mp 2 k \phi \text {. }
$$


We then have $\phi A \xi=0$, which shows that $M$ is a Hopf hypersurface. We next take a principal curvature vector $X$ orthogonal to $\xi$ associated to a principal curvature $\lambda$. Hence, from Lemma A and Equation (4.2) we find that the principal curvature $\lambda$ satisfies one of the following quadratic equations:

$$
4 \lambda^{2}+8 k \lambda+c-4 k \delta=0 \text { or } 4 \lambda^{2}-8 k \lambda+c+4 k \delta=0 .
$$

Since $k$ and $\delta$ are constant, this implies that $\lambda$ is also constant on the connected real hypersurface $M$. Thus we can see that our real hypersurface $M$ is locally congruent to a homogeneous Hopf hypersurface in $\mathbf{C H}(c)$ (see Theorem B). We shall check (4.2) one by one for each homogeneous Hopf hypersurface $M$.

When $M$ is of type $\left(\mathrm{A}_{0}\right)$, we know by the table of the principal curvatures that $A \phi+\phi A=\sqrt{|c|} \phi$.

When $M$ is of type $\left(\mathrm{A}_{1,0}\right)$, we see that $A \phi+\phi A=\sqrt{|c|} \operatorname{coth}(\sqrt{|c|} r / 2) \phi$.

When $M$ is of type $\left(\mathrm{A}_{1,1}\right)$, we have $A \phi+\phi A=\sqrt{|c|} \tanh (\sqrt{|c|} r / 2) \phi$.

When $M$ is of type $\left(\mathrm{A}_{2}\right)$, by the fact that $\phi V_{\lambda_{1}}=V_{\lambda_{1}}$ and $\phi V_{\lambda_{2}}=V_{\lambda_{2}}$ (see Lemma A), we find that our real hypersurface $M$ does not satisfy (4.2).

When $M$ is of type (B), by the fact that $\phi V_{\lambda_{1}}^{0}=V_{\lambda_{2}}^{0}$ and $\phi V_{\lambda_{2}}^{0}=V_{\lambda_{1}}^{0}$ for each radius $r \in(0, \infty)$, where $V_{\lambda_{i}}^{0}=\left\{v \in T M \mid A v=\lambda_{i} v, v \perp \xi\right\}$ for $i=1,2$ (see Lemma A) we can see that our real hypersurface $M$ satisfies $A \phi+\phi A=\left(\lambda_{1}+\lambda_{2}\right) \phi$ with $\lambda_{1}+\lambda_{2}=\sqrt{|c|} \operatorname{coth}(\sqrt{|c|} r)$.

Therefore by virtue of the above discussion we can see that a real hypersurface $M$ satisfies (4.2) if and only if $M$ is of either type $\left(\mathrm{A}_{0}\right)$, type $\left(\mathrm{A}_{1,0}\right)$, type $\left(A_{1,1}\right)$ or type $(B)$. We next investigate the sectional curvatures of these homogeneous real hypersurfaces.

When $M$ is of type $\left(\mathrm{A}_{1,0}\right)$, we take a pair $(X, Y)$ of orthonormal vectors that are orthogonal to $\xi$. In order to estimate the sectional curvature $K$ of $M$, we calculate $K(\sin \theta \cdot X+\cos \theta \cdot \xi, Y)$. It follows from (2.7) that

$$
K(\sin \theta \cdot X+\cos \theta \cdot \xi, Y)=(c / 4)\left\{\sin ^{2} \theta\left(1+3 g(\phi X, Y)^{2}\right)-\operatorname{coth}^{2}(\sqrt{|c|} r / 2)\right\} .
$$

This gives the following inequalities:

$$
c-(c / 4) \operatorname{coth}^{2}(\sqrt{|c|} r / 2) \leqq K \leqq(-c / 4) \operatorname{coth}^{2}(\sqrt{|c|} r / 2) .
$$

We remark that $K(X, \phi X)=c-(c / 4) \operatorname{coth}^{2}(\sqrt{|c|} r / 2)$ and $K(X, \xi)=(-c / 4)$. $\operatorname{coth}^{2}(\sqrt{|c|} r / 2)$ for each unit vector $X$ orthogonal to $\xi$. By easy computation we see that $c-(c / 4) \operatorname{coth}^{2}(\sqrt{|c|} r / 2) \geqq 0$ if and only if $0<r \leqq \log 3 / \sqrt{|c|}$. Hence a geodesic sphere $G(r)$ is of nonnegative sectional curvature at some point $x \in M$ if and only if the radius $r$ satisfies $0<r \leqq \log 3 / \sqrt{|c|}$.

When $M$ is of type $\left(\mathrm{A}_{0}\right)$, by taking $r \rightarrow \infty$ in (4.4) we have $3 c / 4 \leqq K \leqq$ $-c / 4$ at its each point.

When $M$ is of type $\left(\mathrm{A}_{1,1}\right)$, for a unit vector $X$ with $A X=$ $(\sqrt{|c|} / 2) \tanh (\sqrt{|c|} r / 2) X$ from $(2.7)$ we see

$$
K(X, \phi X)=c+\frac{|c|}{4} \tanh ^{2}\left(\frac{\sqrt{|c|} r}{2}\right)<c+\frac{|c|}{4}=\frac{3 c}{4}<0 .
$$


When $M$ is of type (B), by taking a unit vector $X$ orthogonal to $\xi$ with $A X=(\sqrt{|c|} / 2) \operatorname{coth}(\sqrt{|c|} r / 2) X$, from $(2.7)$ we get $K(X, \phi X)=3 c / 4<0$. Thus we have proved the "if" part in our Theorem.

Theorem.

$(\Rightarrow)$ By the above discussion we get easily the "only if" part in our

Thus we have proved Theorem 2 .

We cannot characterize a geodesic sphere $G(r)$ of radius $r$ with $0<r \leqq$ $\log 3 / \sqrt{|c|}$ by the condition that there exists a positive constant $k$ with $k \geqq \sqrt{|c|}$ such that $M$ satisfies either $d \eta(X, Y)=k g(X, \phi Y)$ for all $X, Y \in T M$ or $d \eta(X, Y)$ $=-k g(X, \phi Y)$ for all $X, Y \in T M$. Indeed, we have the following:

THeOrem C ([7]). A real hypersurface $M^{2 n-1}$ of $\mathbf{C} H^{n}(c), n \geqq 2$ satisfies the condition that there exists a positive constant $k$ with $k \geqq \sqrt{|c|}$ such that $M$ satisfies either $d \eta(X, Y)=k g(X, \phi Y)$ for all $X, Y \in T M$ or $\bar{d} \eta(X, Y)=-k g(X, \phi Y)$ for all $X, Y \in T M$ if and only if $M$ is locally congruent to either a geodesic sphere $G(r)$ of radius $r(0<r \leqq \log 3 / \sqrt{|c|})$ or a homogeneous real hypersurface of type (B) of radius $r(0<r \leqq \log 3 /(2 \sqrt{|c|}))$.

\section{5. $\phi$-invariance of the shape operator of real hypersurfaces in a complex hyperbolic space}

In order to guarantee Fact (3), we prove the following:

Proposition 5. There does not exist a real hypersurface $M^{2 n-1}$ with strongly $\phi$-invariant shape operator $A$ in $\mathbf{C} H^{n}(c), n \geqq 2$.

Proof. Suppose that there exists a real hypersurface $M$ with strongly $\phi$-invariant shape operator $A$ in this ambient space. Then the shape operator $A$ of this real hypersurface $M$ satisfies

$$
-\phi A \phi X=A X \text { for all } X \in T M \text {. }
$$

Putting $X=\xi$ in (5.1), we have $A \xi=0$, so that in particular, $M$ is a Hopf hypersurface. This, together with Lemma A(1), implies

$$
2 \lambda A \phi X=\frac{c}{2} \phi X \text { for each vector } X(\perp \xi) \text { with } A X=\lambda X
$$

It follows from (5.1) and (5.2) that $4 \lambda^{2}=c<0$, which is a contradiction. Thus we get the desired conclusion.

Remark 2. We emphasize that there do exist real hypersurfaces $M^{2 n-1}$ with strongly $\phi$-invariant shape operator in $\mathbf{C} P^{n}(c), n \geqq 2$. In $\mathbf{C} P^{n}(c), n \geqq 2$, a real hypersurface $M^{2 n-1}$ has strongly $\phi$-invariant shape operator if and only if 
$M$ is locally congruent to a hypersurface of type (A) with radius $r=\pi /(2 \sqrt{c})$ (see [11]).

We shall prove Theorem 3 .

$(\Rightarrow)$ Since $\phi A=A \phi$ holds on $M$ (see Lemma B), for any $X, Y(\in T M)$ orthogonal to $\xi$ we see

$$
\begin{aligned}
g(A \phi X, \phi Y) & =g(\phi A X, \phi Y)=-g\left(A X, \phi^{2} Y\right) \\
& =-g(A X,-Y+\eta(Y) \xi)=g(A X, Y),
\end{aligned}
$$

which shows that our Hopf hypersurface $M$ has weakly $\phi$-invariant shape operator. Moreover, by the above estimation on sectional curvatures we find that our real hypersurface $M$ is of nonnegative sectional curvatures at its each point. Thus we have shown the "only if" part in our Theorem.

$(\Leftarrow)$ For a unit vector $X$ orthogonal to $\xi$ with $A X=\lambda X$, we find

$$
(2 \lambda-\delta) g(A \phi X, \phi X)=\lambda(2 \lambda-\delta),
$$

which, combined with Lemma A(1), implies that our Hopf hypersurface $M$ has at most three constant principal curvatures $\delta, \lambda_{1}$ and $\lambda_{2}$, where $\lambda_{1}$ and $\lambda_{2}$ are solutions to the quadratic equation $4 \lambda^{2}-4 \delta \lambda-c=0$. Then $M$ is locally congruent to either a hypersurface of type (A) or a homogeneous real hypersurface of type (B) (see Theorem B). But, the principal curvatures $\lambda_{1}$ and $\lambda_{2}$ of every homogeneous real hypersurface of type (B) do not satisfy the above quadratic equation. Hence $M$ is of type (A). Furthermore, by virtue of the estimation on the sectional curvature of hypersurfaces of types $\left(\mathrm{A}_{1,0}\right),\left(\mathrm{A}_{0}\right)$ and $\left(A_{1,1}\right)$ and the following estimation on the sectional curvature of a hypersurface of type $\left(\mathrm{A}_{2}\right)$

$$
K(X, \phi X)=c-\frac{c}{4} \tanh ^{2}\left(\frac{\sqrt{|c|}}{2} r\right)<0 \text { for each unit vector } X \in V_{\lambda_{2}}
$$

we obtain the "if" part in our Theorem. Thus we have proved Theorem 3.

Remark 3. (1) The shape operator of every hypersurface of type (A) in a nonflat complex space form is weakly $\phi$-invariant.

(2) There exist non-Hopf hypersurfaces $M^{2 n-1}$ with weakly $\phi$-invariant shape operator in a nonflat complex space form $\tilde{M}_{n}(c), n \geqq 2$. For example, we take an arbitrary ruled real hypersurface $M$ in this ambient space. Then the shape operator $A$ of $M$ satisfies $g(A X, Y)=0$ for all $X, Y(\perp \xi) \in T M$ (for details, see [13]). Hence this real hypersurface $M$ has weakly $\phi$-invariant shape operator in a trivial sense.

(3) The discussion in the proof of Theorems 2 and 3 shows that geodesic spheres $G(r)(0<r \leqq \log 3 / \sqrt{|c|})$ in $\mathbf{C} H^{n}(c)$ are the only examples of hypersurfaces of type (A) all of whose sectional curvatures are nonnegative in this ambient space (cf. [10]). 


\section{Contact metric structures and the proof of Proposition 1}

Let $M^{2 n-1}(n \geqq 2)$ be a differentiable manifold with an almost contact metric structure $(\phi, \xi, \eta, g)$. That is, this structure satisfies the following equations:

$$
\phi^{2} X=-X+\eta(X) \xi, \quad \phi \xi=0, \quad \eta(\xi)=1, \quad g(\phi X, \phi Y)=g(X, Y)-\eta(X) \eta(Y)
$$

for all vectors $X$ and $Y$ on $M$. We say $M$ to be a Sasakian manifold if the structure tensor $\phi$ satisfies the differential equation

$$
\left(\nabla_{X} \phi\right) Y=g(X, Y) \xi-\eta(Y) X
$$

for all tangent vectors $X, Y \in T M$, where $\nabla$ denotes the Riemannian connection related to the metric $g$ of $M$. A Sasakian manifold is called a Sasakian space form of constant $\phi$-sectional curvature $c$ if the sectional curvature $K(u, \phi u):=$ $g(R(u, \phi u) \phi u, u)$ satisfies $K(u, \phi u)=c$ for each unit vector $u$ orthogonal to $\xi$. The following is a Sasakian analogue of Schur's Theorem.

THEOREM C. If the $\phi$-sectional curvature at each point of a Sasakian manifold $M$ of dimension $\geqq 5$ does not depend on the choice of $\phi$-section at that point, then it is constant on $M$. The curvature tensor of $M$ is given by

$$
\begin{aligned}
R(X, Y) Z= & \frac{c+3}{4}\{g(Y, Z) X-g(X, Z) Y\} \\
& +\frac{c-1}{4}\{\eta(X) \eta(Z) Y-\eta(Y) \eta(Z) X+g(X, Z) \eta(Y) \xi-g(Y, Z) \eta(X) \xi \\
& \quad+g(Z, \phi Y) \phi X-g(Z, \phi X) \phi Y+2 g(X, \phi Y) \phi Z\},
\end{aligned}
$$

where $c$ is the constant $\phi$-sectional curvature of $M$.

For the standard construction of Sasakian space forms, see pp. 114-115 in [6]. The following is the unique existence theorem of Sasakian space forms.

THEOREM D. For any two simply connected complete Sasakian manifolds of constant $\phi$-sectional curvature $c$, there exists an isomorphism between them which preserves their almost contact metric structures.

We here review the construction of Sasakian space forms of constant $\phi$-sectional curvature $c(<1)$ from the viewpoint of submanifold theory. The following lemma is essentially due to Berndt [3].

Lemma C. Let $M^{2 n-1}$ be a connected real hypersurface isometrically immersed into $\mathbf{C H}^{n}(c), n \geqq 2$. Suppose that $M$ is a Sasakian manifold with respect to one of the almost contact metric structures $(\phi, \xi, \eta, g)$ and $(\phi,-\xi,-\eta, g)$ induced from the Kähler structure $(J, g)$ of $\mathbf{C} H^{n}(c)$. Then $M$ is locally congruent to one of the following homogeneous real hypersurfaces of $\mathbf{C H}^{n}(c)$ : 
i) $A$ horosphere in $\mathbf{C H}{ }^{n}(-4)$;

ii) $A$ geodesic sphere $G(r)$ of radius $r(0<r<\infty)$ with $\tanh (\sqrt{|c|} r / 2)=$ $\sqrt{|c|} / 2$ in $\mathbf{C H}^{n}(c)(-4<c<0)$

iii) A tube of radius $r(0<r<\infty)$ around a totally geodesic $\mathbf{C} H^{n-1}(c)$ with $\tanh (\sqrt{|c|} r / 2)=2 / \sqrt{|c|}$ in $\mathbf{C H}^{n}(c)(c<-4)$.

In these cases, $M$ has constant $\phi$-sectional curvature $c+1$.

Proof. Suppose that our real hypersurface $M$ is a Sasakian manifold. Then it follows from (2.3) and (6.1) that

$$
g(X, Y) \xi-\eta(Y) X=\eta(Y) A X-g(A X, Y) \xi
$$

for all vectors $X, Y \in T M$. Setting $X=Y=\xi$ in Equation (6.2), we find that $\xi$ is principal. So we can choose another principal curvature vector $u$ orthogonal to $\xi$. Then, putting $Y=\xi$ in Equation (6.2), we see that

$$
A u=-u \text { for each vector } u \text { perpendicular to } \xi \text {. }
$$

This, together with Lemma A, Theorem B and the above table of the principal curvature vectors, implies that our real hypersurface $M$ is locally congruent to one of i), ii) and iii). Here, we change the unit normal vector $\mathscr{N}$ into $-\mathscr{N}$ on each real hypersurface $M$ in the list of Theorem B. Note that the shape operator $A$ of our Sasakian real hypersurface $M$ satisfies $A=-I+(c / 4) \eta \otimes \xi$. This, combined with (2.6), shows that the $\phi$-sectional curvature $K(u, \phi u)$ on our real hypersurface $M$ satisfies $K(u, \phi u)=c+1$ for every unit vector $u$ orthogonal to $\xi$.

Conversely, we take a real hypersurface $M$ which is locally congruent to one of i), ii) and iii). Then, without loss of generality we can see that the shape operator $A$ of our real hypersurface $M$ is of the form $A=-I+(c / 4) \eta \otimes \xi$. This, together with (2.3), yields (6.1), so that $M$ is a Sasakian manifold.

We shall prove Proposition 1 . We consider a geodesic sphere $G$ of radius $r(0<r \leqq \log 3 / \sqrt{|c|})$ in $\mathbf{C H}^{n}(c)$. Then we have

$$
0<\tanh \left(\frac{\sqrt{|c|}}{2} r\right) \leqq \tanh \left(\frac{\log 3}{2}\right)=\frac{1}{2},
$$

which, together with Equation $\tanh (\sqrt{|c|} r / 2)=\sqrt{|c|} / 2$ in Lemma C(ii), implies $-1 \leqq c<0$. Thus by virtue of Lemma $\mathrm{C}$ we obtain the desired statement of Proposition 1 .

\section{REFERENCES}

[1] T. Adachi, M. Kameda and S. Maeda, Geometric meaning of Sasakian space forms from the viewpoint of submanifold theory, Kodai Math. J. 33 (2010), 383-397.

[2] T. Adachi, S. Maeda And M. Yamagishi, Length spectrum of geodesic spheres in a non-flat complex space form, J. Math. Soc. Japan 54 (2002), 373-408. 
[3] J. BERndT, Real hypersurfaces with constant principal curvatures in complex space forms, Geometry and topology of submanifolds II, Avignon, 1988, World Sci. Publ. Teaneck, NJ, 1990, 10-19.

[4] J. BERNDT, Real hypersurfaces with constant principal curvatures in complex hyperbolic space, J. Reine Angew. Math. 395 (1989), 132-141.

[5] J. Berndt and H. TAmaru, Cohomogeneity one actions on noncompact symmetric spaces of rank one, Trans. Amer. Math. Soc. 359 (2007), 3425-3438.

[6] D. E. BlaIR, Riemannian geometry of contact and symplectic manifolds, Progress in math. 203, Birkhäuser, 2002.

[7] Y. H. KIM AND S. MAEDA, Real hypersurfaces in a nonflat complex space form and their almost contact metric structures, Colloquium Math. 124 (2011), 117-131.

[8] M. KimuRA AND S. MAEDA, Sectional curvatures of some homogeneous real hypersurfaces in a complex projective space, Topics in differential geometry, complex analysis and mathematical physics, World Sci., 2006, 196-204.

[9] U-H. Ki AND Y. J. SuH, On real hypersurfaces of a complex space form, Math. J. Okayama Univ. 32 (1990), 207-221.

[10] S. MaEdA, Nonnegatively curved geodesic spheres in a complex hyperbolic space, C.R. Math. Rep. Acad. Sci. Canada 34 (2013), 114-120.

[11] S. Maeda And H. Naitoh, Real hypersurfaces with $\phi$-invariant shape operator in a complex projective space, Glasgow Math. J. 53 (2011), 347-358.

[12] Y. MAEDA, On real hypersurfaces of a complex projective space, J. Math. Soc. Japan 28 (1976), 529-540.

[13] R. Niebergall and P. J. Ryan, Real hypersurfaces in complex space forms, Tight and taut submanifolds (T. E. Cecil and S. S. Chern, eds.), Cambridge Univ. Press, 1998, 233-305.

[14] R. TAKagi, On homogeneous real hypersurfaces in a complex projective space, Osaka J. Math. 10 (1973), 495-506.

[15] Y. Tashiro and S. Tachibana, On Fubinian and $C$-Fubinian manifolds, Kōdai Math. Sem. Rep. 15 (1963), 176-183.

Tetsuo Kajiwara

Department of Mathematics

SAGa University

1 HonZyo, SAGa 840-8502

JAPAN

E-mail: tkajiwara1009@yahoo.co.jp

Sadahiro Maeda

Department of Mathematics

SAGa University

1 Honzyo, SAGa, 840-8502

JAPAN

E-mail: smaeda@ms.saga-u.ac.jp 